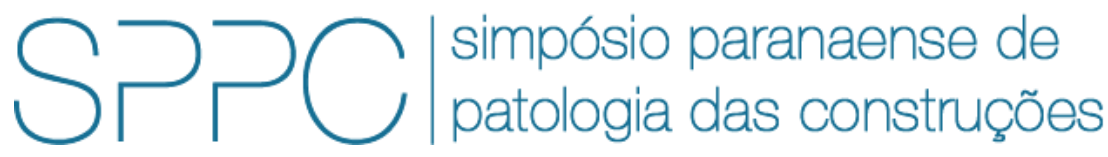

ISSN 2526-7248 artigo n. 2SPPC1035, pp. 421-434, 2017

\title{
Monitoramento de reação álcali agregado em barras de concreto instrumentadas por sensores de Bragg em diferentes condições de exposição
}

\author{
Mário Sergio Mandú Kleina', Mariana D’Orey Gaivão Portella Bragança², Kleber Franke Portella ${ }^{3}$, \\ Bruno Endo Ribeiro ${ }^{4}$, Emerson Alberti ${ }^{5}$ \\ ${ }^{1}$ Mestrando, PPGECC UFPR/LACTEC, mario.kleina@lactec.org.br \\ 2 Doutora, LACTEC, mariana.portella@lactec.org.br \\ ${ }^{3}$ Doutor, LACTEC, portella@lactec.org.br \\ ${ }^{4}$ Graduando, LACTEC, bruno.ribeiro@lactec.org.br \\ ${ }^{5}$ Mestre, ELEJOR, emerson@elejor.com.br
}

\begin{abstract}
Resumo: A reação álcali agregado consiste, basicamente, em uma reação química entre os minerais potencialmente reativos constituintes de agregados e os hidróxidos que estão dissolvidos na pasta de cimento e tende a provocar a deterioração grave de estruturas de concreto, devido às suas manifestações patológicas, relacionadas à fissuração em mapa, expansão, e consequente deslocamento dos elementos estruturais. O monitoramento da reação, em laboratório, se dá pelo acompanhamento das deformações de corpos de prova, a partir de ensaios normatizados. No entanto, o acompanhamento de tais reações ocorrentes em estruturas de grande porte ainda representa um desafio, principalmente no que tange o monitoramento não destrutivo. Esforços mecânicos longitudinais e variações nas condições ambientais provocam alterações no comprimento de onda de Bragg, o que permite o uso destas redes como sensores de deformação e de temperatura. Neste trabalho, foi proposto o monitoramento da deformação linear de barras de concreto, preparadas com agregados potencialmente reativos, instrumentadas por sensores de Bragg para a avaliação da ocorrência de reação álcali agregado. O monitoramento das deformações foi realizado por 15 meses e complementado com a caracterização microestrutural e química elementar do concreto.
\end{abstract}

Palavras-chave: Concreto; Fibra óptica; Reação álcali agregado; Instrumentação.

Abstract: The alkali aggregate reaction consists of a chemical reaction between the potentially reactive minerals constituent of aggregates and the hydroxides that are dissolved in the cement paste. It tends to cause serious deterioration of concrete structures due to their pathological manifestations related to cracking, expansion, and consequent displacement of the structural elements. The diagnosis of this reaction in the laboratory is developed by the deformations monitoring of test specimens, from standardized tests. However, the observation of such reactions occurring in large structures still presents a challenge, especially regarding non-destructive monitoring. Longitudinal mechanical stresses and variations in environmental conditions cause changes in the Bragg wavelength, which allows the use of these networks as strain and temperature sensors. In this work, it was proposed the monitoring of linear deformation of concrete bars, prepared with potentially reactive aggregates, instrumented by Bragg sensors for the evaluation of the occurrence of aggregate alkali reaction. The deformation monitoring was carried out for 15 months and complemented with the microstructural characterization of the concrete.

Keywords: Concrete; Optical fiber; Alkali aggregate reaction; Instrumentation. 


\section{Introdução}

Sensores de fibra óptica apresentam um elevado potencial como um meio não destrutivo para avaliar a condição de estruturas de concreto. Em contraste com as técnicas de avaliação não destrutivas existentes, as fibras ópticas são capazes de detectar variações mínimas nas condições estruturais por meio de medições remotas. O monitoramento permite a detecção do início e do progresso de degradações mecânicas ou ambientais em estruturas de concreto [1].

As redes de Bragg (FBG) são estruturas periódicas de modulação do índice de refração, ' $n$ ', no núcleo da fibra óptica, ao longo do eixo de propagação da luz. A rede opera como um filtro espectral reflexivo que seleciona um comprimento de onda de uma banda larga que tenha sido emitida na fibra. Este comprimento de onda se relaciona com a periodicidade espacial da rede (Figura 1) [2]. Após a incidência desta banda larga de comprimentos de onda, o sinal emitido é dividido em transmitido e refletido, com a passagem pela rede de Bragg, sendo o último conhecido como comprimento de Bragg, conforme apresentado na Figura 2 [3].

$$
\lambda_{B}=2 n \Lambda
$$

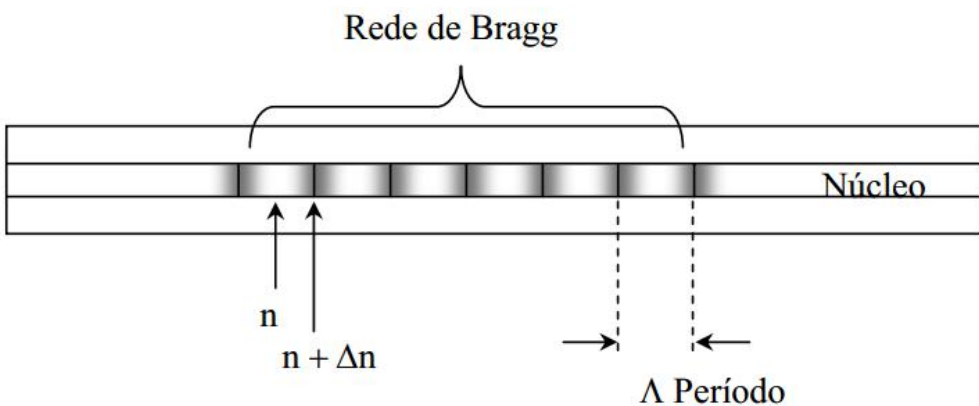

Figura 1: Expressão geral da condição de Bragg em que o comprimento de onda refletido, $\lambda_{B}$, está relacionado com o passo espacial da modulação do índice de refração, $\Lambda$, e com o índice de refração efetivo do núcleo, $n$. [4-5] 


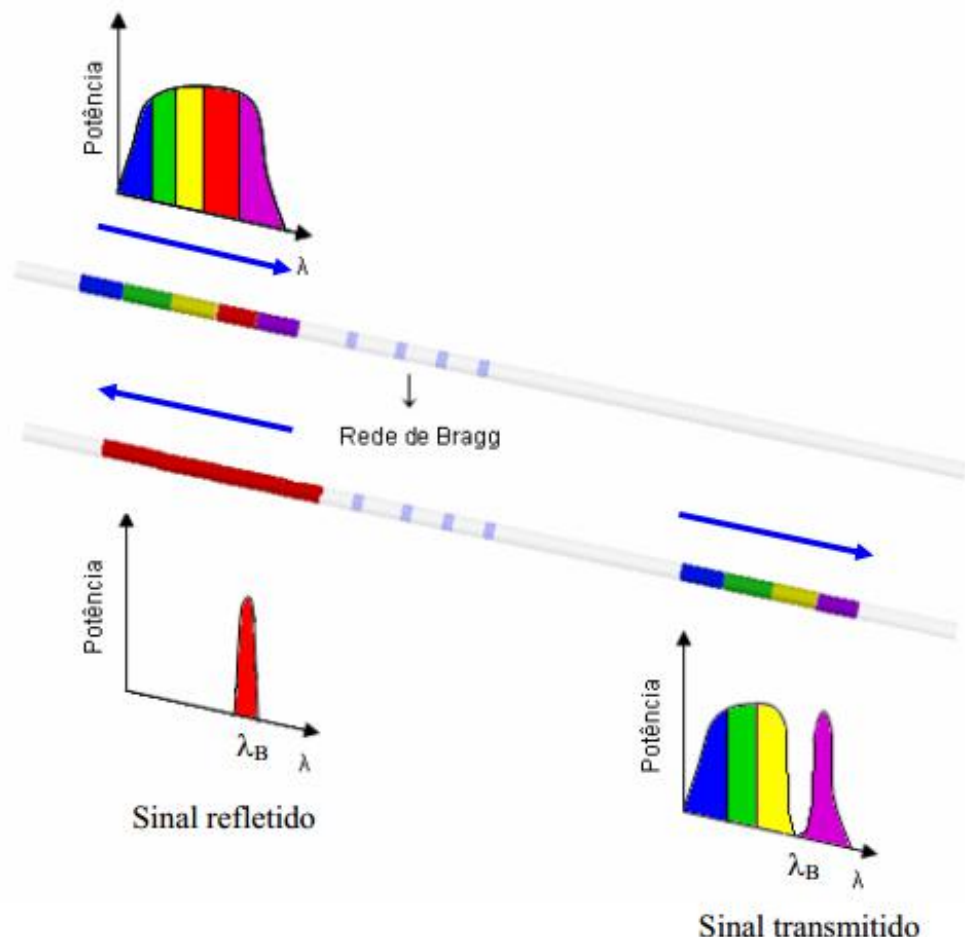

Figura 2: Desenho esquemático do princípio do funcionamento de uma FBG.[3]

Esforços mecânicos longitudinais e variações de temperatura provocam alterações no comprimento de onda de Bragg. A variação deste comprimento de onda é o que permite o uso destas redes como sensores de deformação e de temperatura [2]

A reação álcali agregado (RAA) é um dos fenômenos mais deletérios que ocorrem em estruturas de concreto. Consiste, basicamente, em uma reação química entre os minerais potencialmente reativos constituintes de agregados e os hidróxidos que estão dissolvidos na pasta de cimento e na solução de poros de concreto [6].

O produto da reação é um material de silicato amorfo, com características de gel, cuja expansão pode provocar a fissuração da matriz cimentícia e do agregado, o que conduz a uma deterioração grave da estrutura [7]. A reação manifesta-se no concreto por um padrão de fissuração em mapa, expansão, e consequente deslocamento dos elementos estruturais, acompanhada de desagregação de fragmentos da superfície [8].

Dentre os fatores que mais influenciam na RAA, podem-se mencionar os seguintes grupos de agentes [9]:

- Agentes relacionados ao meio ambiente, como a umidade, a temperatura e os ciclos de molhagem e secagem;

- Agentes químicos, como a presença de sais e aditivos;

- Agentes associados ao concreto, relacionados ao fator água/cimento, ao teor de álcalis e à dimensão das partículas dos agregados; e

- Agentes que controlam a reação e a expansão, como as adições pozolânicas, o teor de ar incorporado, a porosidade dos agregados e os aditivos químicos inibidores da expansão. 
No caso da temperatura, sua elevação acelera a RAA e, consequentemente, a formação do gel, pois aumenta a solubilidade da sílica e diminui a do $\mathrm{Ca}(\mathrm{OH})_{2}$, 0 que provoca o aumento da taxa de reação. De modo geral, sob condições de temperaturas elevadas, as reações químicas tendem a ocorrer com uma velocidade maior, o que não é diferente nas reações que ocorrem nas estruturas de concreto. 0 aumento da temperatura torna o gel menos viscoso e por esse motivo, pode percolar com mais facilidade nos vazios do concreto [10]

O monitoramento da RAA, em laboratório, se dá pelo acompanhamento das deformações de corpos de prova de argamassa e concreto, a partir de ensaios normatizados pelo conjunto de normas NBR 15577 [13-18]. No entanto, o acompanhamento de tais reações ocorrentes em estruturas de grande porte, como pontes e barragens de energia, ainda representa um desafio, principalmente no que tange o monitoramento não destrutivo.

Assim, neste trabalho, foi proposto o monitoramento da deformação linear de barras de concreto, preparadas com agregados potencialmente reativos, instrumentadas por sensores FBG ("Fiber Bragg Grating"), de modo a avaliar, comparativamente, a exposição em ambiente controlado e submetidas ao intemperismo natural.

\section{Materiais e métodos}

\subsection{Sensores FBG}

Neste trabalho, foram utilizados sensores da marca Sylex, modelo ASC-01, com comprimento de ancoragem de $1000 \mathrm{~mm}$ e precisão de $0,005 \mathrm{~mm}$. O sistema de âncoras permitiu que os sensores fossem incorporados ao concreto de modo a captar as deformações causadas por mudanças de temperatura e esforços mecânicos por meio da variação dos comprimentos de onda. Essas variações foram comparadas ao comprimento verificado no momento da instalação e após o pré tracionamento do sensor. Na Figura 3 está ilustrado o sensor utilizado no monitoramento das deformações.

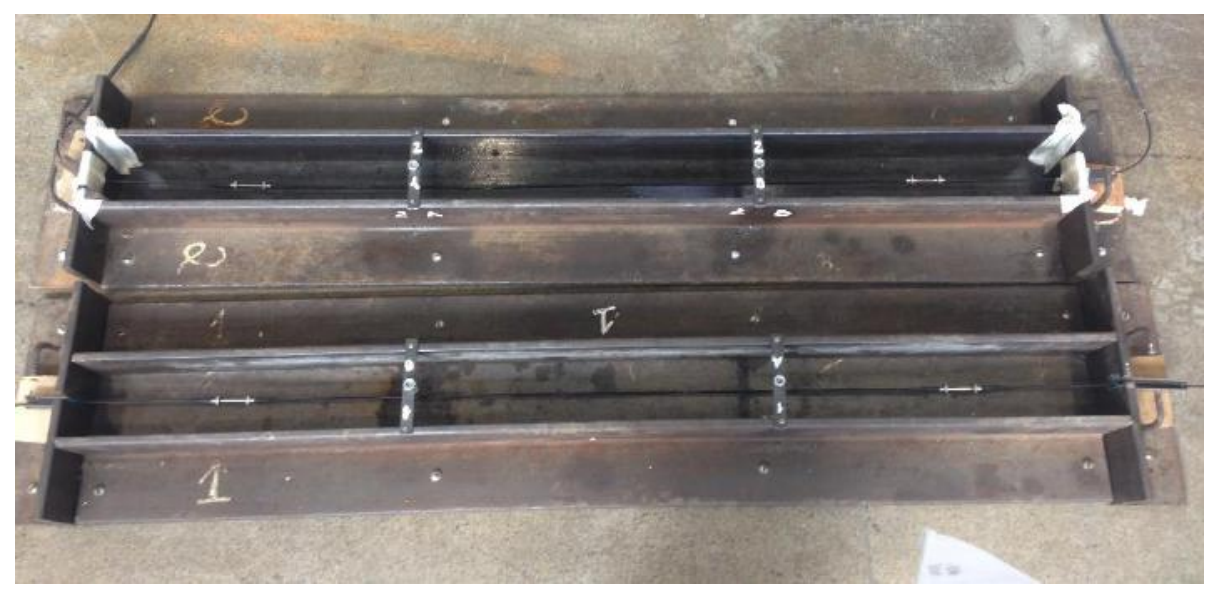

Figura 3: Sensor Sylex ASC-01 devidamente posicionado na altura média das barras antes do lançamento do concreto.

Por meio da base de dados ("datasheet") de cada um dos sensores, fornecida pelo fabricante, permitiu-se realizar a subtração dos efeitos da variação de temperatura 
para as barras expostas em ambiente externo. De acordo com a Sylex, a deformação relativa entre o sistema de ancoragem dos sensores ( $\mu \varepsilon$ ) é dada pela fórmula apresentada na Equação 1 , onde: $\lambda_{0, \text { inst,strain }}$ é o comprimento de onda no instante da instalação do sensor; $\lambda_{\text {act,strain }}$ é o comprimento de onda no instante da leitura; $T_{0, \text { inst }}$ é a temperatura no momento da instalação do sensor; $T_{a c t}$ é a temperatura no instante da leitura; e $A$ e $B$ são as constantes de calibração fornecidas pelo fabricante.

$$
\Delta \varepsilon=\frac{\frac{\lambda_{\text {act }, \text { strain }}-\lambda_{0, \text { inst }, \text { strain }}}{\lambda_{0, \text { inst } \text { strain }}}-B .\left(T_{\text {act }}-T_{0, \text { inst }}\right)}{A}
$$

\subsection{Sistema de aquisição de dados}

O interrogador SCN-84 S-line Scan 800 da marca Sylex, acoplado a um multiplexador de canais (Figura 4), compatível com os sensores, foi utilizado no monitoramento. Este módulo tem capacidade para o monitoramento simultâneo de 4 canais. O software S-line Sentinel 1.0, fornecido pelo fabricante juntamente com 0 interrogador, possui uma interface simples permitindo a coleta, análise e avaliação dos dados.

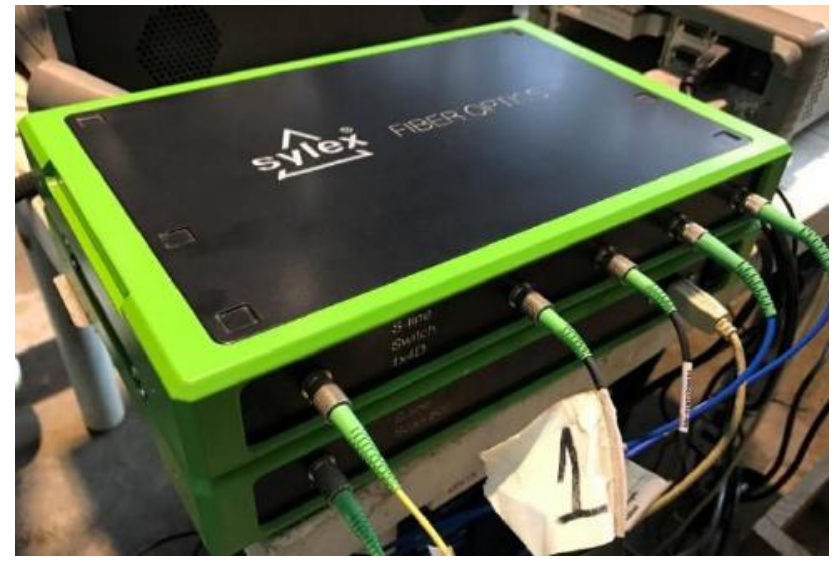

Figura 4: Interrogador SCN-84 S-line Scan 800 da marca Sylex, utilizado para a aquisição dos dados durante o monitoramento.

\subsection{Moldagem das barras instrumentadas}

Os concretos estudados foram dosados utilizando dois traços extremos, relativos ao consumo de cimento, a exemplo do que é aplicado em obras de infraestrutura (como barragens, pontes e reservatórios), conforme apresentado na Tabela 1. Os insumos utilizados, também de acordo com o aplicável em grandes obras de engenharia, foram o cimento Portland CP IV-32, um agregado basáltico com reatividade potencial, britado em granulometrias de areia e brita 25, e a água de abastecimento público. Os insumos foram previamente caracterizados por espectroscopia de fluorescência de raios-X (XRF), em amostras de pastilha fundida, analisada no equipamento Espectrofotômetro PANalytical Axios 55 Max (cimento) e por metodologia normativa (agregados), conforme apresentadas na Tabela 2. 
Tabela 1: Dados dos traços de concreto utilizados $-1 \mathrm{~m}^{3}$.

\begin{tabular}{c|c|c}
\hline \multirow{2}{*}{ Materiais } & \multicolumn{2}{|c}{ Massa (kg) } \\
\cline { 2 - 3 } & $\mathbf{E}-3339$ & $\mathbf{E}-3340$ \\
\hline Cimento CP IV - 32 & 458 & 255 \\
\hline Areia de britagem produzida em laboratório & 871 & 1077 \\
\hline Brita 9,5/25,0 produzida em laboratório & 962 & 964 \\
\hline Relação água/cimento & 0,45 & 0,70 \\
\hline $\begin{array}{c}\text { Abatimento do concreto conforme NBR NM 67 } \\
{[11](\mathrm{mm})}\end{array}$ & 60 & 75 \\
\hline
\end{tabular}

Tabela 2: Metodologias utilizadas para caracterização dos materiais utilizados.

\begin{tabular}{c|c}
\hline Materiais & Metodologia de caracterização \\
\hline Cimento CP IV 32 & Espectroscopia de fluorescência de raios X (XRF) \\
\hline Agregados & Análise petrográfica - NBR 7389-2 [12] \\
\hline
\end{tabular}

Foram confeccionadas quatro barras, sendo duas para cada traço, com dimensões de $(100 \times 100 \times 1400) \mathrm{mm}$. As barras foram moldadas em duas camadas e adensadas manualmente de modo a permitir a instalação dos sensores FGB, posicionados entre as camadas, conforme apresentado na Figura 5. Os sensores foram posicionados e pré-tracionados em $10 \%$ do limite de deslocamento, conforme recomendação do fabricante, permitindo leituras referentes à esforços de tração e compressão.

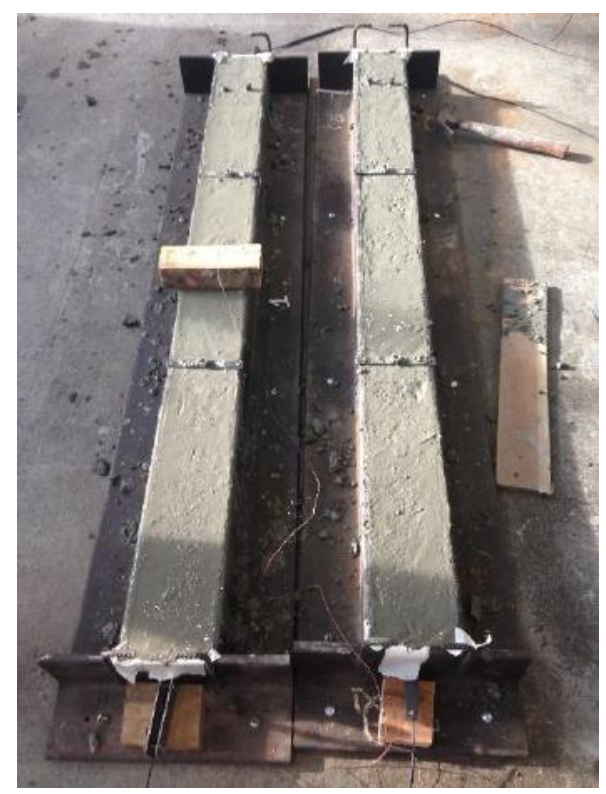

Figura 5: Barras recém moldadas e devidamente instrumentadas pelos sensores FBG. 
Realizou-se a cura das barras, com a manutenção de um filme de água durante 7 dias e, após esse período, foram divididas em duas condições de envelhecimento: mantidas totalmente imersas, em um tanque com água à temperatura constante de $(40 \pm 2)^{\circ} \mathrm{C}$ (a exemplo da norma NBR 15577 [16]) e expostas em ambiente externo, sujeitas ao intemperismo natural, conforme apresentado na Figura 6. Os corpos de prova instrumentados foram monitorados continuamente com relação à deformação linear pelos sensores FGB, por um período de 15 meses.

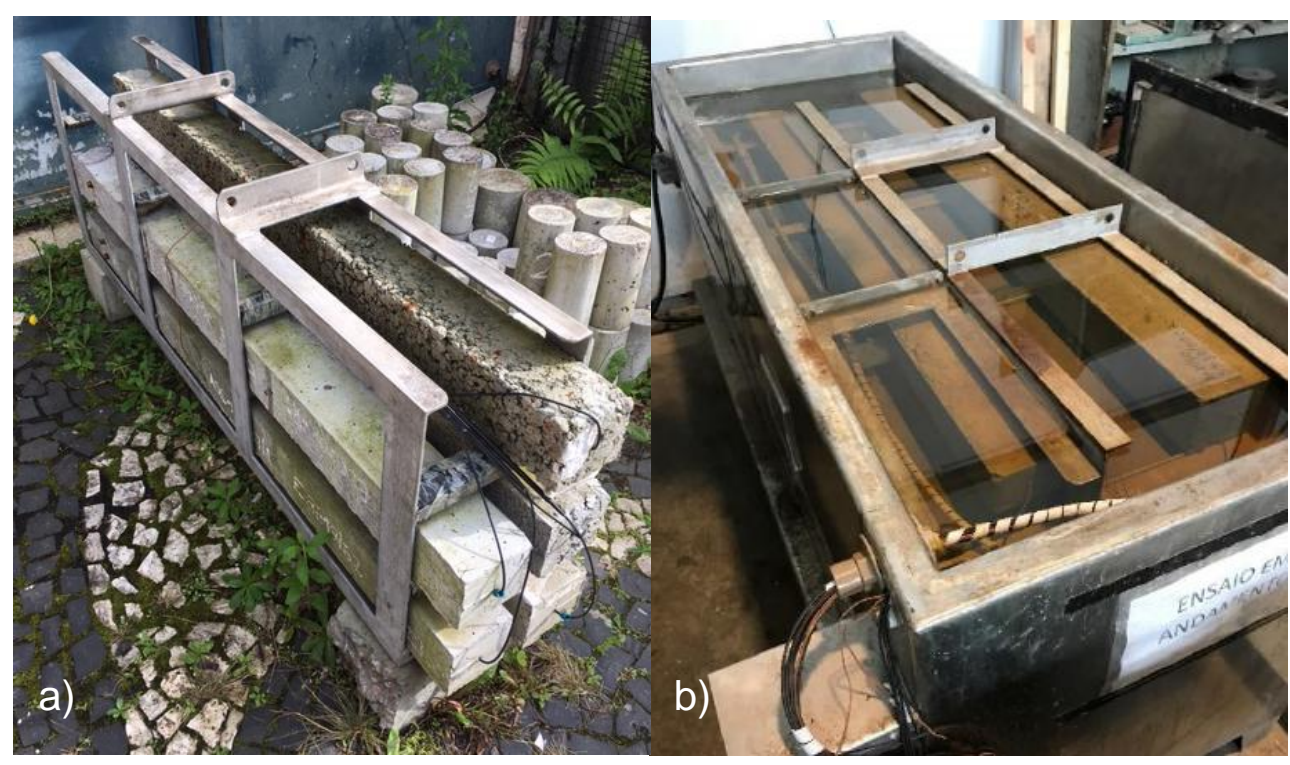

Figura 6: Barras monitoradas (a) expostas ao intemperismo natural e (b) mantidas à temperatura constante de $(40 \pm 2) \stackrel{\circ}{ } \mathrm{C}$.

\subsection{Caracterização microestrutural dos concretos}

Após os 15 meses de exposição, foram realizadas microanálises em amostras de superfície de fratura, retiradas das barras prismáticas de concreto, sem tratamento prévio de cobertura para o aumento de sua condutividade (in natura). Para a observação, foi utilizado o microscópio eletrônico de varredura, tipo FEG ("field emission gun") com monocristal de tungstênio e camada de zircônio, modelo MIRA3 LM, com uso dos detectores de elétrons secundários (SE) e de elétrons retroespalhados (BSE), marca TESCAN. Os parâmetros do equipamento seguiram com a tensão de corrente do feixe de elétrons de $15 \mathrm{kV}$, intensidade do feixe ("beam intensity") 18,00, abertura do feixe ("spot size") de 18,0 nm, distância de trabalho ("work distance"-WD) de $15 \mathrm{~mm}$ e modo de resolução de scanner.

A avaliação qualitativa e semi-quantitativa dos elementos químicos presentes nos materiais, procedeu $\mathrm{o}$ uso do detector de espectrometria de raios- $\mathrm{X}$, marca OXFORD INSTRUMENTS.

\section{Resultados e discussão}

\subsection{Caracterização dos materiais}

Os resultados de caracterização do cimento CP IV 32, por espectroscopia de fluorescência de raios-X (XRF), estão apresentados na Tabela 3. Para os 
agregados, pela análise petrográfica, estes foram caracterizados como uma rocha basáltica, potencialmente reativa devido à presença de argilomineral.

Tabela 3. Caracterização do cimento CP IV 32 utilizado, através da XRF.

\begin{tabular}{c|c|c|c|c}
\hline $\mathrm{CaO} \%$ & $\mathrm{SiO}_{2} \%$ & $\mathrm{Na}_{2} \mathrm{O} \%$ & $\mathrm{Al}_{2} \mathrm{O}_{3} \%$ & $\mathrm{MgO} \%$ \\
\hline 45,18 & 28,44 & 1,31 & 10,28 & 3,21 \\
\hline $\mathrm{Fe}_{2} \mathrm{O}_{3} \%$ & $\mathrm{SO}_{3} \%$ & $\mathrm{TiO}_{2} \%$ & Outros $\%$ & Perda ao Fogo \% \\
\hline 3,69 & 2,43 & -- & 2,21 & 3,25 \\
\hline
\end{tabular}

\subsection{Monitoramento das barras instrumentadas}

Na Figura 7 e Figura 8 estão apresentados os gráficos, correspondentes aos valores de variação dimensional, verificados nos 2 traços de concretos moldados, para cada um dos estudos de envelhecimentos propostos.

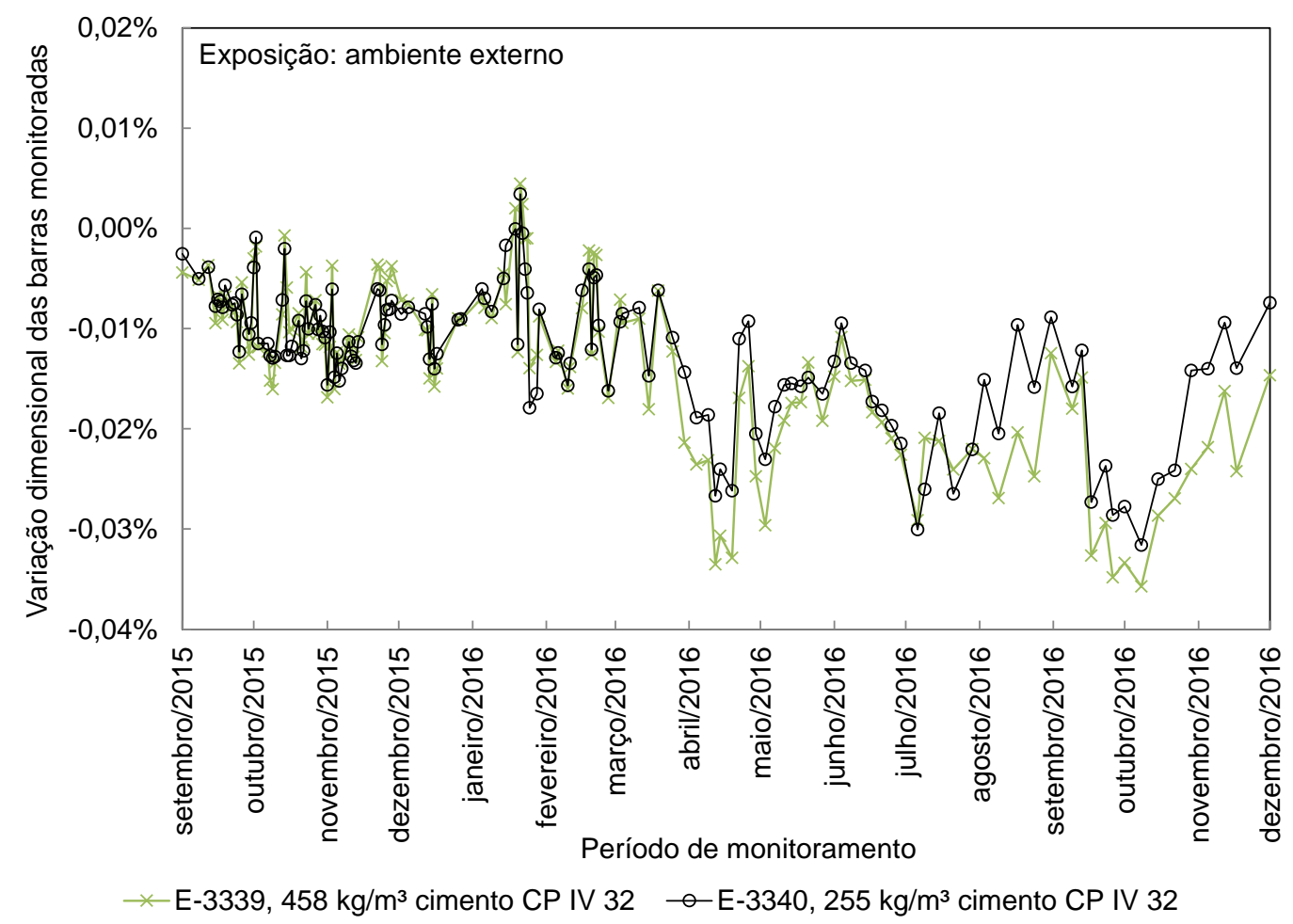

Figura 7: Dados de monitoramento das barras expostas ao intemperismo natural durante 15 meses. 


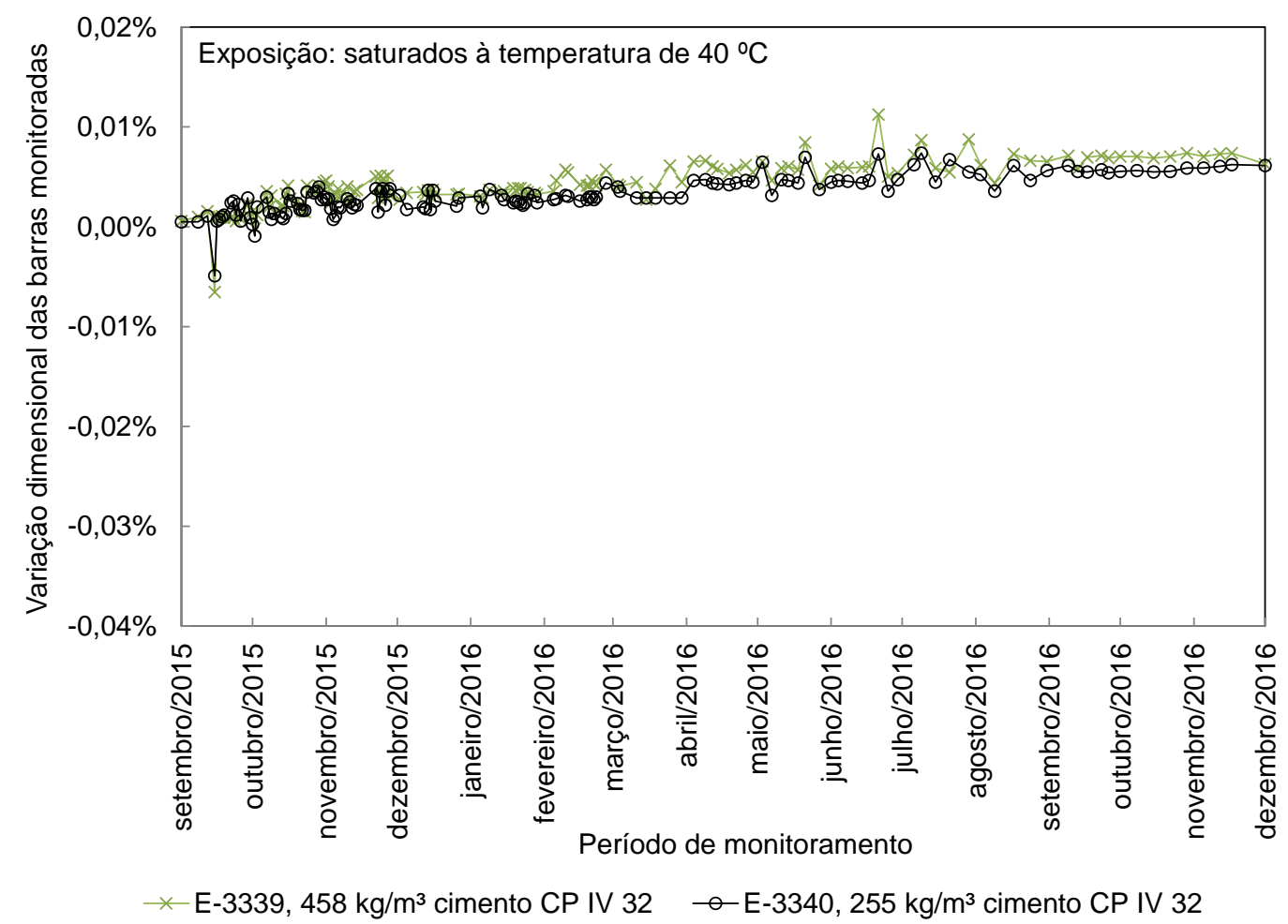

Figura 8: Dados de monitoramento das barras mantidas à temperatura constante durante 15 meses.

Conforme pode ser verificado no gráfico da Figuras 7, a análise da expansão resultante das barras em ambiente externo, ao longo do período monitorado, mostrou que ambos os traços de concreto vêm apresentando um processo de contração volumétrica. Tal fenômeno pode ser decorrente da retração térmica, devido à inferior disponibilidade de água ou até pela ocorrência de lixiviação dos produtos solúveis formados, causadas pela exposição à ciclos de molhagem e secagem no concreto.

Já, para as amostras mantidas na temperatura constante de $(40 \pm 2){ }^{\circ} \mathrm{C}$, foi observado o processo expansivo, de acordo com o gráfico da Figura 8. Estatisticamente, ambos têm mostrado expansões equivalentes para o período, porém, com uma maior tendência para o traço de maior consumo de cimento. Muito embora a qualidade do concreto com maior consumo de cimento tende a ser superior, a maior disponibilidade de álcalis na solução de poros (cerca de $44 \%$, em massa), tende a favorecer a ocorrência da reação, conforme já citado por Wang [9]. Tal fato evidenciou a ineficácia do maior consumo de cimento na dosagem dos diferentes traços a fim de mitigar possíveis reações deletérias.

Quando analisadas as diferentes condições de exposição, em comparação, verificou-se que as barras expostas ao intemperismo natural apresentaram uma variação maior das deformações, porém, a exposição à temperatura constante foi mais severa ao concreto, pelo fato de proporcionar um ambiente mais favorável à formação de subprodutos expansivos.

Os resultados das análises efetuadas por MEV-EDS de amostras das barras de concreto, conforme apresentado nas Figuras 9 a 12, corroboraram com aqueles obtidos pelos dados de monitoramento. Foi verificada a presença de subprodutos de RAA e a ocorrência de cristais com morfologia de agulhas aciculares e composição 
química similar a etringita (sulfoaluminatos de cálcio), principalmente no interior dos poros e nas interfaces entre a pasta de cimento e os agregados, para todas as amostras, com maior evidência naquelas mantidas à temperatura constante.

Especificamente para as barras mantidas em exposição natural, foi observada a ocorrência de microfissuração dos agregados (Figura 11), com deposição de um gel sílico-cálcico, o que leva a crer a ocorrência inicial da reação, muito embora ainda não tenham sido observadas as variações dimensionais. Isso pode estar correlacionado com a suposta lixiviação dos produtos solúveis formados, proporcionada pelos ciclos de umedecimento e secagem aos quais estes materiais estavam sujeitos.

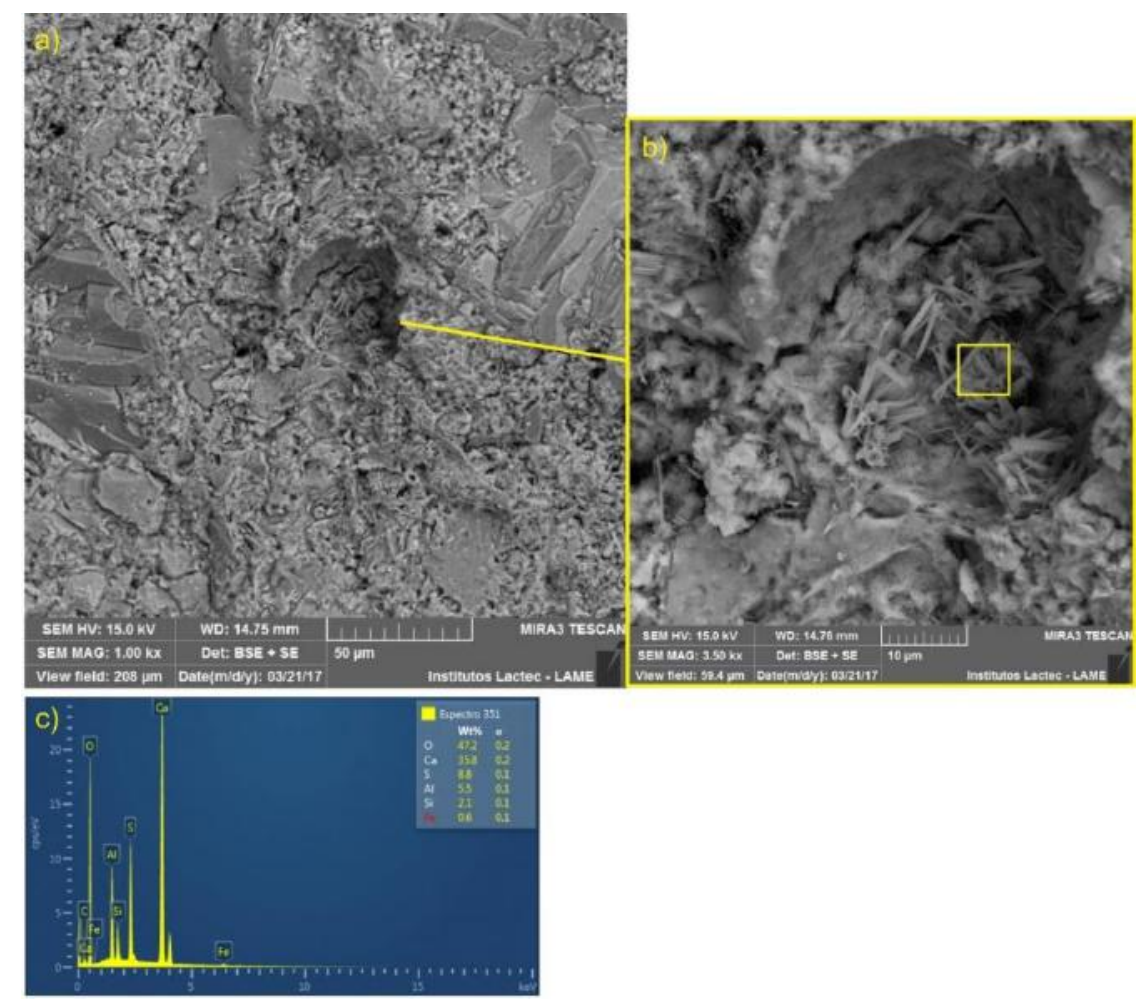

Figura 9: Micrografia da amostra E-3360 (exposta ao ambiente externo) evidenciando o preenchimento dos poros com a formação de subprodutos (a), detalhe da formação dos subprodutos (b), análise química elementar contendo a formação de sulfoaluminato de cálcio, possivelmente expansivo (c). 

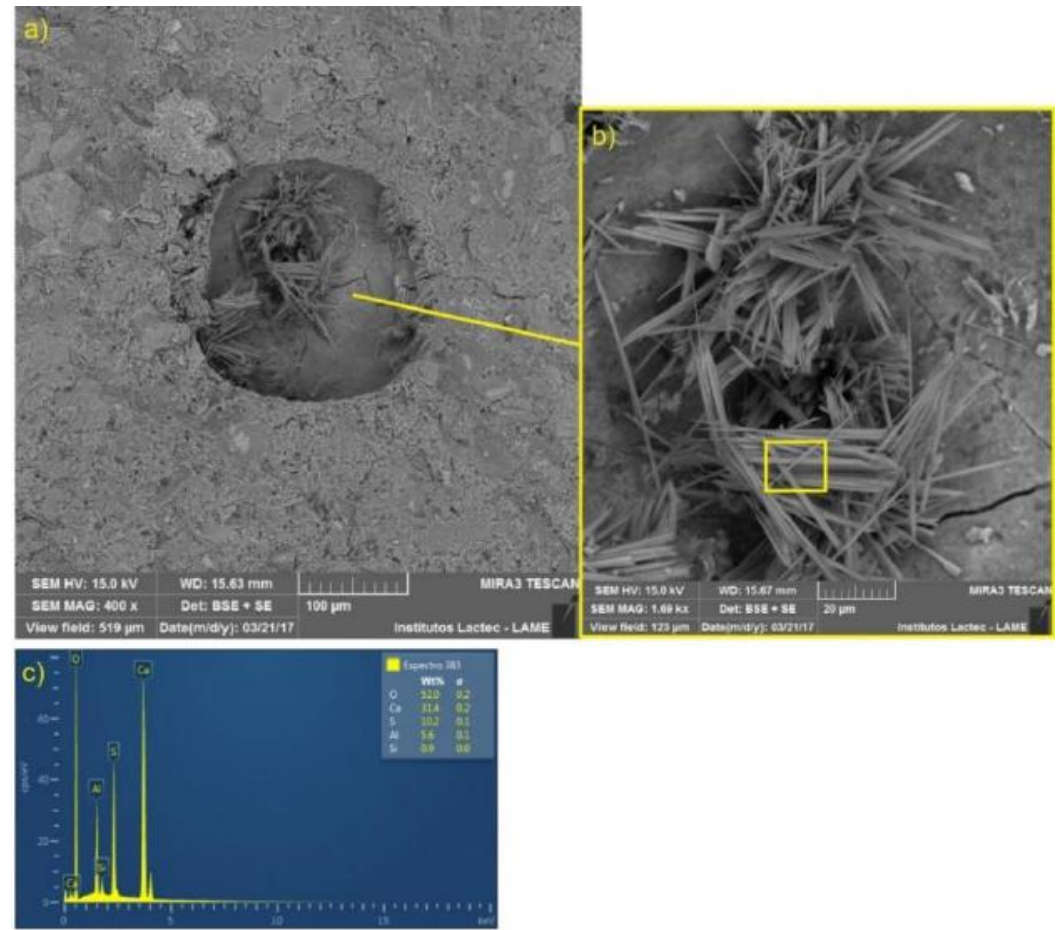

Figura 10: Micrografia da amostra E-3360 (imersa em água com temperatura controlada de $40^{\circ} \mathrm{C}$ ) evidenciando o preenchimento dos poros com a formação de subprodutos (a), detalhe da formação dos subprodutos (b), análise química elementar contendo a formação de sulfoaluminato de cálcio, possivelmente expansivo (c).
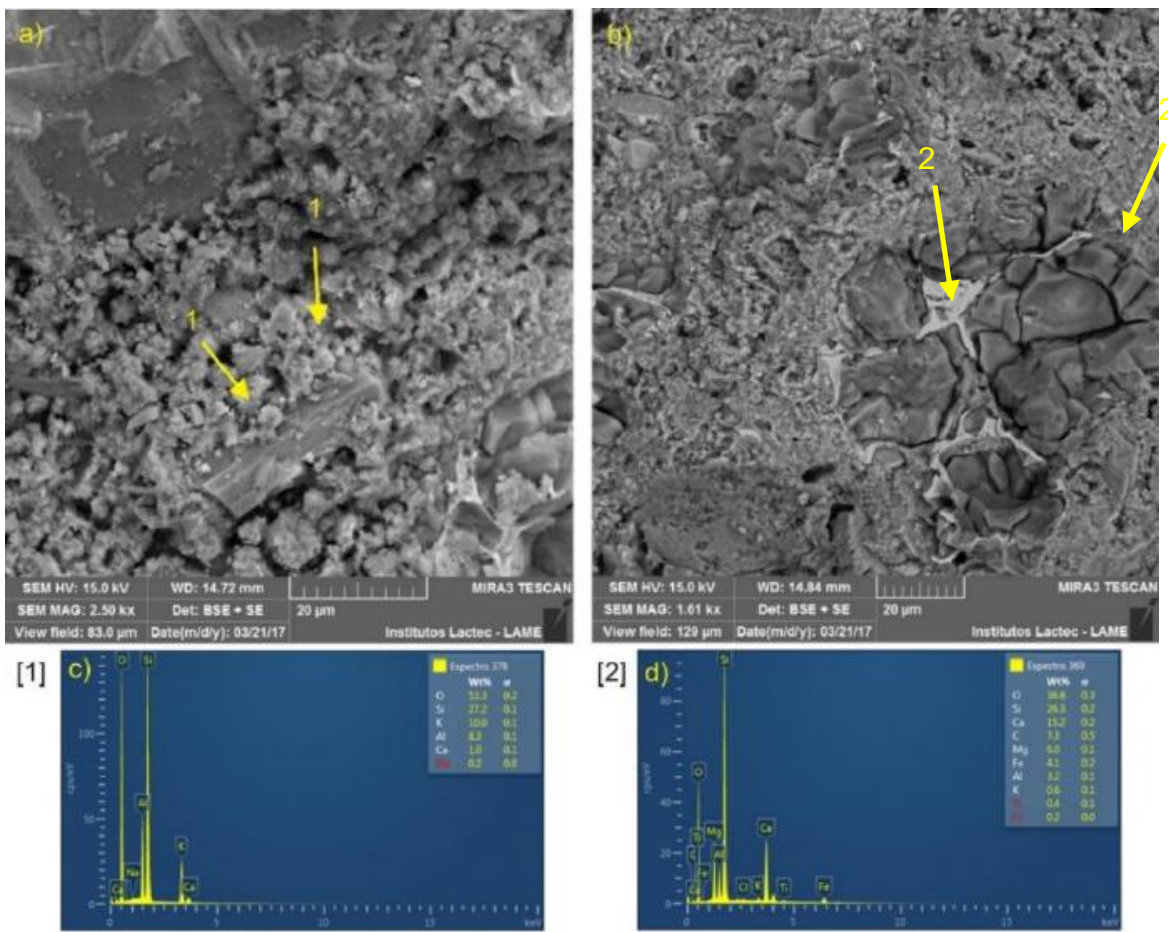

Figura 11: Micrografia da amostra E-3361 (exposta ao ambiente externo) evidenciando a formação de subprodutos na interface agregado/pasta de cimento (a) e (b), análise química elementar contendo a possível formação do gel de RAA (c) e (d). 

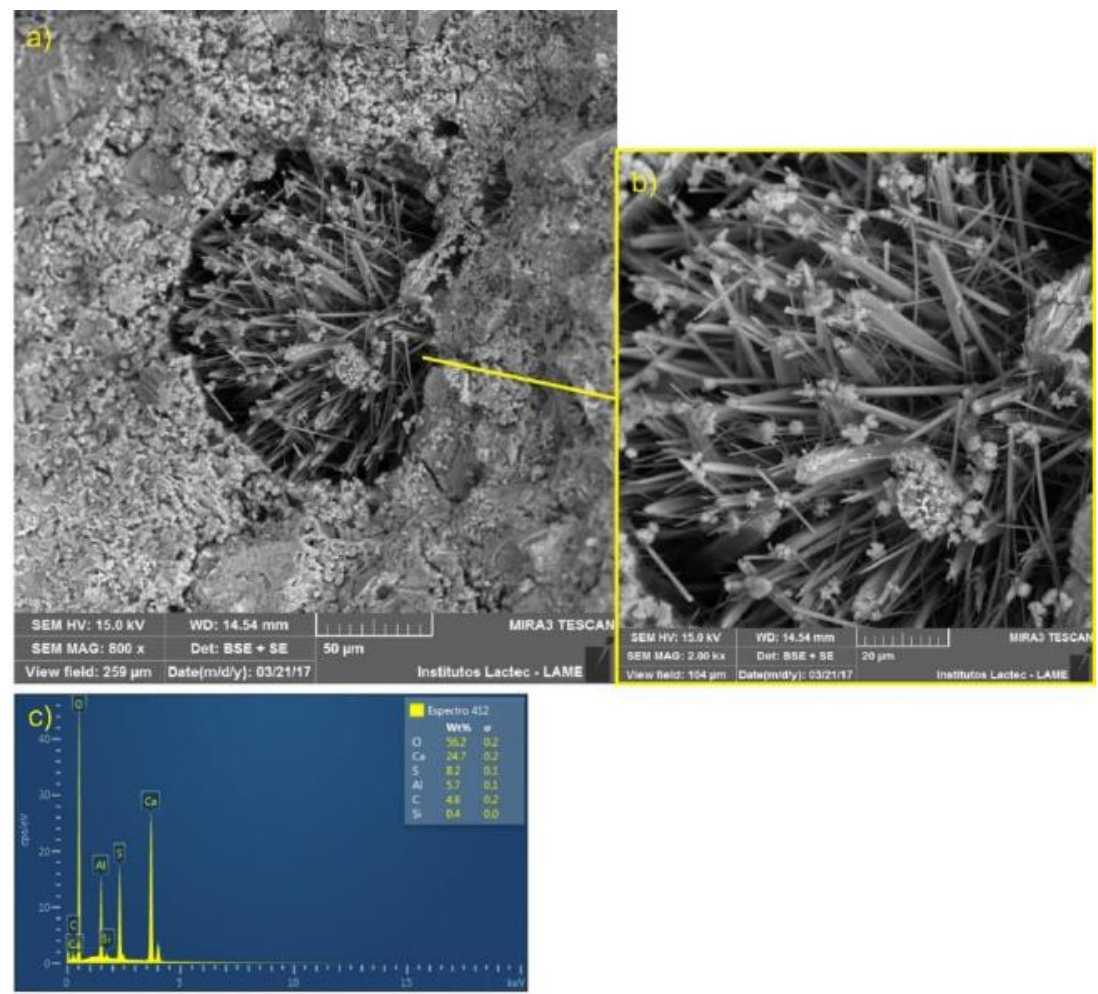

Figura 12: Micrografia da amostra E-3361 (imersa em água com temperatura controlada $-40^{\circ} \mathrm{C}$ ) evidenciando o preenchimento dos poros com a formação de subprodutos (a), detalhe da formação dos subprodutos (b), análise química elementar contendo a formação de sulfoaluminato de cálcio, possivelmente expansivo (c).

\section{Conclusão}

O monitoramento da ocorrência de expansões devido à reação álcali agregado em barras de concreto por meio de sensores ópticos FBG se mostrou um método nãodestrutivo eficaz para a detecção do início e do progresso de tais manifestações patológicas. Foi possível verificar a ocorrência de expansões, logo para os primeiros meses de monitoramento, e correlacioná-las à formação de subprodutos de reação, pela análise microestrutural e química elementar. Comparando-se as diferentes condições de exposição, verificou-se que à temperatura constante foi mais severa ao concreto, pelo fato de proporcionar um ambiente mais favorável à formação de subprodutos expansivos. Tais resultados evidenciaram que o desenvolvimento destas metodologias pode ser ampliado para a escala de monitoramento de grandes estruturas civis, no sentido de detectar variações físico-químicas na qualidade dos concretos, antevendo a necessidade de reparos ou manutenções.

\section{Agradecimentos}

Os autores agradecem à infraestrutura e ao apoio em recursos humanos e financiamento à ELEJOR, projeto P\&D 2945-0004/2013, à ANEEL, aos Institutos LACTEC, ao CNPq Lei 8010/90 (LI 15/2187214-1; LI 14/4695814-5; LI14/34107269), ao CNPq/PIBIT e de bolsa DT, processo 302672/2016-8*, e à Universidade Federal do Paraná - PPGECC/UFPR. 


\section{Referências}

[1] Ansari, F. (1997) State-of-the-art in the applications of fiber-optic sensors to cementitious composites. Cement and Concrete Composites. USA.

[2] Erdorgan, T. Fiber gratin spectra. Journal of Lightwave Technology, v. 15, n. 8, 1997.

[3] Hill, K. O. \& Meltz, G., Fiber Gragg Gratings Technology: Fundamentals and Overview, Journal of Lightwave Technology, v. 15, n. 8, 1997.

[4] Hill, K. O., Fuji, Y., Johnson, D. C. \& Kawasaki, B. S., Photosensitivity in Optical Waveguides: Application to Reflection Filter Fabrication, Appl. Phys. Lett, Vol. 32, 1978.

[5] OTHONOS, A., KALLI, K., PUREUR, D. \& MUGNIER, A.; "Fiber Bragg Grating: Fundamentals and Applications, in Telecommunications and Sensing", London, Artech House, 1999.

[6] Sanchez, L.F.M. (2008). Contribuição ao estudo dos métodos de ensaio na avaliação das reações álcali-agregado em concretos. Dissertação (Mestrado), Universidade de São Paulo, Departamento de engenharia de construção civil, São Paulo. 168p.

[7] Tambelli, C.E., Schneider, J.F., Hasparyk, N.P., \& Monteiro, P.J.M. (2006). Study of the structure of alkali-silica reaction gel by high-resolution NMR spectroscopy. Journal of Non-Crystalline Solids, 352, pp. 3429-3436.

[8] Silveira, A.A. (2007). Contribuição ao estudo do efeito da casca de arroz em concretos submetidos à reação álcali-agregado. Tese (Doutorado), Universidade Federal do Rio Grande do Sul, Porto Alegre. 226 p.

[9] Wang, H. (1990). Alkali-silica reaction: mechanism. Significance of chemical and mineral admixtures. Tese (Doutorado), University of Calgary, Calgary Canadá. 330 p.

[10] Hasparyk, N.P. (1999). Investigação dos mecanismos da reação álcaliagregado - efeito da cinza da casca de arroz e da sílica ativa. Dissertação (Mestrado), Universidade Federal de Goiás, Escola de Engenharia Civil, Goiânia. 257 p.

[11] NBR NM 67 (1998). Concreto - Determinação da consistência pelo abatimento do tronco de cone. Associação Brasileira de Normas Técnicas - ABNT, Rio de Janeiro, Brasil.

[12] NBR 7389-2 (2009). Agregados - Análise petrográfica de agregado para concreto. Parte 2: Agregado graúdo. Associação Brasileira de Normas Técnicas - ABNT, Rio de Janeiro, Brasil.

[13] NBR 15577-1 (2008). Agregados - Reatividade álcali-agregado Parte 1: Guia para avaliação da reatividade potencial e medidas preventivas para uso de agregados em concreto. Associação Brasileira de Normas Técnicas - ABNT, Rio de Janeiro, Brasil.

[14] NBR 15577-2 (2008). Agregados - Reatividade álcali-agregado Parte 2: Coleta, preparação e periodicidade de ensaios de amostras de agregados para 
concreto. Associação Brasileira de Normas Técnicas - ABNT, Rio de Janeiro, Brasil.

[15] NBR 15577-3 (2008). Agregados - Reatividade álcali-agregado Parte 3: Análise petrográfica para verificação da potencialidade reativa de agregados em presença de álcalis do concreto. Associação Brasileira de Normas Técnicas ABNT, Rio de Janeiro, Brasil.

[16] NBR 15577-4 (2008). Agregados - Reatividade álcali-agregado Parte 4: Determinação da expensão em barras de argamassa pelo método acelerado. Associação Brasileira de Normas Técnicas - ABNT, Rio de Janeiro, Brasil.

[17] NBR 15577-5 (2008). Agregados - Reatividade álcali-agregado Parte 5: Determinação da mitigação da expansão em barras de argamassa pelo método acelerado. Associação Brasileira de Normas Técnicas - ABNT, Rio de Janeiro, Brasil.

[18] NBR 15577-6 (2008). Agregados - Reatividade álcali-agregado Parte 6: Determinação da expansão em prismas de concreto o. Associação Brasileira de Normas Técnicas - ABNT, Rio de Janeiro, Brasil. 